\title{
Identification of a novel oncogene, $M M S 22 L$, involved in lung and esophageal carcinogenesis
}

\author{
MINH-HUE NGUYEN ${ }^{1}$, KOJI UEDA $^{1,4}$, YUSUKE NAKAMURA $^{1}$ and YATARO DAIGO ${ }^{1-3}$ \\ ${ }^{1}$ Laboratory of Molecular Medicine, Human Genome Center, Institute of Medical Science, \\ The University of Tokyo, Tokyo; ${ }^{2}$ Department of Medical Oncology and ${ }^{3}$ Cancer Center, \\ Shiga University of Medical Science, Otsu; ${ }^{4}$ Laboratory for Biomarker Development, \\ Center for Genomic Medicine, RIKEN Yokohama Institute, Yokohama, Japan
}

Received April 27, 2012; Accepted June 12, 2012

DOI: $10.3892 /$ ijo.2012.1589

\begin{abstract}
Genome-wide gene expression profile analyses using a cDNA microarray containing 27,648 genes or expressed sequence tags identified MMS22L (methyl methanesulfonate-sensitivity protein 22-like) to be overexpressed in the majority of clinical lung and esophageal cancers, but not expressed in normal organs except testis. Transfection of siRNAs against $M M S 22 L$ into cancer cells suppressed its expression and inhibited cell growth, while exogenous expression of MMS22L enhanced the growth of mammalian cells. MMS22L protein was translocated to the nucleus and stabilized by binding to $\mathrm{C}$-terminal portion of NFKBIL2 [nuclear factor of kappa (NFKB) light polypeptide gene enhancer in B-cells inhibitor-like 2]. Expression of a C-terminal portion of NFKBIL2 protein including the MMS22L-interacting site in cancer cells could reduce the levels of MMS22L in nucleus and suppressed cancer cell growth. Interestingly, reduction of MMS22L by siRNAs in cancer cells inhibited the TNF- $\alpha$-dependent activation of RelA/p65 in the NFKB pathway and expression of its downstream anti-apoptotic molecules such as Bcl-XL and TRAF1. In addition, knockdown of MMS22L expression also enhanced the apoptosis of cancer cells that were exposed to DNA-damaging agents including 5-FU and CDDP. Our data strongly suggest that targeting MMS22L as well as its interaction with NFKBIL2 could be a promising strategy for novel cancer treatments, and also improve the efficacy of DNA damaging anticancer drugs.
\end{abstract}

\section{Introduction}

Lung cancer is the most common cause of cancer-related death, and the worldwide annual death by lung cancer was

Correspondence to: Professor Yataro Daigo, Department of Medical Oncology, Shiga University of Medical Science, Seta Tsukinowa-cho, Shiga 520-2192, Otsu, Japan

E-mail: ydaigo@ims.u-tokyo.ac.jp

Key words: MMS22L, oncogenes, therapeutic target, lung cancer, esophageal cancer estimated to be 1.3 million (1). Esophageal squamous cell carcinoma (ESCC) is one of the most common gastrointestinal tract cancers in Asian countries (2). Although a huge body of knowledge about the biology of lung or esophageal carcinogenesis has been accumulated, the development of novel cancer therapeutics remains inefficient to improve patients with these cancers (3). In fact, in spite of development of various molecular targeted therapies, a limited proportion of patients can receive clinical benefit from them (4).

Through genome-wide gene expression analysis of lung and esophageal cancers, we have isolated a number of oncogenes that were involved in the development and/or progression of cancer (5-41). Among the genes upregulated in these cancers, we focused on MMS22L (methyl methanesulfonate-sensitivity protein 22-like) which is highly expressed in the majority of clinical lung and esophageal cancers. Our original gene expression profile database also revealed that this gene is highly expressed in clinical cervical cancers, but scarcely expressed in normal tissues except testis, suggesting that $M M S 22 L$ encodes a cancer-testis antigen that can be defined by predominant expression in various types of cancer and undetectable expression in normal tissues except germ cells in testis or ovary (4). Cancer-testis antigens are considered to be good candidate molecular targets for developing new therapeutic strategies for cancers.

Constitutive activation of the NFKB pathway is involved in some forms of cancer such as leukemia, lymphoma, colon cancer and ovarian cancer as well as inflammatory diseases (42-45). The main mechanism of this pathway is reported to be the inactivation of $\mathrm{I} \kappa \mathrm{B}$ proteins by mutations as well as amplifications and rearrangements of genes encoding the NFKB transcription factor subunits (42-45). However, more commonly it is thought that changes in the upstream pathways that lead to NFKB activation are likely to be aberrantly upregulated in cancer cells (45). Recently some reports suggested that MMS22L-NFKBIL2 interaction could be essential for genomic stability and homologous recombination in immortalized cell lines, suggesting MMS22L to be a new regulator of DNA replication in human cells (46-49). However, no study has indicated critical roles of activation of MMS22L and NFKBIL2 in clinical cancers and investigated their functional importance in carcinogenesis. Here, we report that MMS22L is involved 
in NFKB pathway in cancer cells through its interaction with NFKBIL2 and might be a promising target for development of novel cancer therapy.

\section{Materials and methods}

Cell lines and clinical samples. The 12 human lung-cancer cell lines used for in this study included nine NSCLC cell lines (A549, NCI-H1373, LC319, NCI-H1781, PC-14, NCI-H358, NCI-H2170, NCI-H520 and LU61) and three small-cell lung cancer (SCLC) cell lines (SBC-3, SBC-5 and DMS114). The 9 human esophageal carcinoma cell lines used in this study were as follows: eight SCC cell lines (TE1, TE3, TE8, TE9, TE10, TE12, TE13 and TE15) and one adenocarcinoma (ADC) cell line (TE7). A cervical cancer cell line HeLa was also included in the study. All cells were grown in monolayers in appropriate media supplemented with $10 \%$ fetal calf serum (FCS) and were maintained at $37^{\circ} \mathrm{C}$ in an atmosphere of humidified air with $5 \% \mathrm{CO}_{2}$. Human airway epithelial cells, SAEC (Cambrex Bio Science Inc.), were also included in the panel of the cells used in this study. Primary lung and esophageal cancer samples had been obtained earlier with informed consent (5-10). This study and the use of all clinical materials mentioned were approved by individual institutional ethics committees.

Semiquantitative RT-PCR. We prepared appropriate dilutions of each single-stranded cDNA prepared from mRNAs of clinical lung and esophageal cancer samples, taking the level of $\beta$-actin $(A C T B)$ expression as a quantitative control. The primer sets for amplification were as follows: $A C T B-\mathrm{F}$ (5'-GAGGTGATAGCA TTGCTTTCG-3') and ACTB-R (5'-CAAGTCAGTGTACAGG TAAGC-3') for ACTB, $M M S 22 L-F$ (5'-GTCTCACCTTGGAC AGATGG-3') and $M M S 22 L-\mathrm{R}$ (5'-CCAAGGATCCTATTACA CAGTTGC-3') for MMS22L. All reactions involved initial denaturation at $95^{\circ} \mathrm{C}$ for $5 \mathrm{~min}$ followed by 22 (for $A C T B$ ) or 30 (for $M M S 22 L$ ) cycles of $95^{\circ} \mathrm{C}$ for $30 \mathrm{sec}, 56^{\circ} \mathrm{C}$ for $30 \mathrm{sec}$, and $72^{\circ} \mathrm{C}$ for $60 \mathrm{sec}$ on a GeneAmp PCR system 9700 (Applied Biosystems).

Northern blot analysis. Human multiple-tissue northern blots (16 normal tissues including heart, brain, placenta, lung, liver, skeletal muscle, kidney, pancreas, spleen, thymus, prostate, testis, ovary, small intestine, colon, leukocyte; BD Biosciences Clontech) were hybridized with a ${ }^{32} \mathrm{P}$-labeled PCR product of $M M S 22 L$. The partial-length cDNA of $M M S 22 L$ was prepared by RT-PCR using primers $M M S 22 L$-F1 (CTGGAAGAGGCA GTTGAAAA) and $M M S 22 L-\mathrm{R} 1$ (ATCGCCCAATATACTG CTCA). Prehybridization, hybridization, and washing were performed according to the supplier's recommendations. The blots were autoradiographed with intensifying screens at $-80^{\circ} \mathrm{C}$ for 7 days.

Anti-MMS22L antibody. Synthesized peptide with the amino acids sequence of CLGQMGQDEMQRLENDNT [1227-1243] (Cysteine was added to the N-terminal) was inoculated into rabbits; the immune sera were purified on affinity columns according to standard methodology. Affinity-purified antiMMS22L antibodies were used for western blot as well as immunocytochemical analyses. We confirmed that the antibody was specific to MMS22L on western blots using lysates from cell lines that had been transfected with MMS22L expression vector as well as those from lung and esophageal cancer cell lines that endogenously expressed MMS22L or not.

Western blot analysis. Cells were lysed in lysis buffer; $50 \mathrm{mM}$ Tris-HCl (pH 8.0), $150 \mathrm{mM} \mathrm{NaCl}, 0.5 \%$ NP-40, $0.5 \%$ deoxycholate-Na, $0.1 \%$ SDS, plus protease inhibitor (Protease Inhibitor Cocktail Set III; Calbiochem). We used ECL western blot analysis system (GE Healthcare Bio-Sciences), as described previously (11).

Immunocytochemical analysis. Cultured cells were washed twice with PBS(-), fixed and rendered permeable in 1:1 acetone: methanol solution for $10 \mathrm{~min}$ at $-20^{\circ} \mathrm{C}$. Prior to the primary antibody reaction, cells were covered with blocking solution [5\% bovine serum albumin in PBS(-)] for 10 min to block non-specific antibody binding. After the cells were incubated with a rabbit polyclonal antibody to human MMS22L (generated to synthesized peptide MMS22L; please see above) or a mouse monoclonal antibody to human NFKBIL2 (Abnova), the Alexa Fluor 488-labelled donkey anti-rabbit secondary antibody (Molecular Probes) or Alexa Fluor 594-labbelled donkey anti-mouse secondary antibody (Molecular Probes) was added to detect endogenous MMS22L or NFKBIL2, individually. Nuclei were stained with 4',6-diamidino-2-phenylindole (DAPI). The antibody-stained cells were viewed with a laser-confocal microscope (TSC SP2 AOBS; Leica Microsystems).

RNA interference assay. Two independent siRNA oligonucleotides against $M M S 22 L$ were designed using the $M M S 22 L$ sequences (GenBank accession no: NM198468). Each siRNA $(600 \mathrm{pM})$ was transfected into two NSCLC cell lines, LC319 and A549 or a cervical cancer cell line HeLa using $30 \mu 1$ of lipofectamine 2,000 (Invitrogen) following the manufacturer's protocol. The transfected cells were cultured for seven days. Cell numbers and viability were measured by Giemsa staining and 3-(4,5-dimethylthiazol-2-yl)-2,5-diphenyltetrazolium bromide (MTT) assay in triplicate (cell counting kit-8 solution; Dojindo Laboratories). The siRNA sequences used were as follows: control-1 (si-LUC: luciferase gene from Photinus pyralis), 5'-CGUACGCGGAAUACUUCGA-3'; control-2 (CNT: On-TARGETplus siControl non-targeting siRNAs of a pool of four oligosnucleotides: 5'-UGGUUUACAUGUCGACUAA-3'; 5'-UGGUUUACAUGUUUUCUGA-3'; 5'-UGGUUUACAUG UUUUCCUA-3'; and 5'-UGGUUUACAUGUUGU GUGA-3'); siRNA-MMS22L-\#1 (si-MMS22L-\#1: 5'-CCGCCAAUAUCA UCUCUAAUU-3'); siRNA-MMS22L-\#2 (si-MMS22L-\#2: 5'-GAA CCUGCAAUACAUGGUAUU-3'). Downregulation of endogenous MMS22L expression in the cell lines by siRNAs for $M M S 22 L$, but not by controls, was confirmed by semiquantitative RT-PCR and western blot analyses.

Cell growth assay. COS-7 or HEK293 cells that express endogenous $M M S 22 L$ at a very low level were transfected with mock or MMS22L-expressing vectors (pCAGGSn-3xFlag-MMS22L) using lipofectamine 2,000 transfection reagent (Roche). Transfected cells were incubated in the culture medium containing $0.8 \mathrm{mg} / \mathrm{ml}$ neomycin (Geneticin, Invitrogen) for 7 days. Expression of MMS22L as well as viability and colony numbers of cells were evaluated by western blot analysis, and MTT and colony-formation assays at day 7. 
Flow cytometric analysis. Cells transfected with siRNA oligonucleotides against $M M S 22 L$ or control siRNAs were plated at densities of $5 \times 10^{5}$ per $60-\mathrm{mm}$ dish. Cells were collected in PBS, and fixed in $70 \%$ cold ethanol for $30 \mathrm{~min}$. After treatment with $100 \mu \mathrm{g} / \mathrm{ml}$ RNase (Sigma-Aldrich), the cells were stained with $50 \mu \mathrm{g} / \mathrm{ml}$ propidium iodide (Sigma-Aldrich) in PBS. Flow cytometric analysis was done on a Cell Lab Quanta SC (Beckman Coulter) and analyzed by CXP Analysis software (Beckman Coulter). The cells selected from at least 10,000 ungated cells were analyzed for DNA content.

\section{Results}

Expression of MMS22L in lung and esophageal cancers. We previously performed genome-wide expression profile analysis of 120 lung cancer cases using microarray consisting of 27,648 cDNAs or ESTs (5-10). Among the genes upregulated in lung and esophageal cancers, we identified $M M S 22 L$ transcript to be frequently overexpressed in lung and esophageal cancers, and confirmed by semiquantitative RT-PCR experiments its elevated expression in all of eleven clinical lung cancers and in four clinical esophageal cancers, although its expression was not detectable in adjacent normal lung and esophagus tissues (Fig. 1a). We further confirmed by western blot analysis high levels of endogenous MMS22L protein in 11 of 12 lung cancer cell lines and in all of 9 esophageal cancer cell lines using anti-MMS22L antibody (Fig. 1b). Northern blot analysis of 16 normal tissues confirmed that $M M S 22 L$ was hardly detectable in normal tissues except the testis (Fig. 1c).

Growth effect of MMS22L. To investigate the relevance of MMS22L to the growth and/or survival of cancer cells, we knocked down the expression of endogenous $M M S 22 L$ in two lung cancer cell lines, LC319 and A549, by means of the RNAi technique using siRNA oligonucreotide for $M M S 22 L$. Semiquantitative RT-PCR experiments detected significant reduction of MMS22L expression in the cells transfected with siRNAs against $M M S 22 L$ (si-\#1 and si-\#2), but not in those with control siRNAs (si-LUC and si-CNT) (Fig. 2a). Colony formation and MTT assays clearly demonstrated that the viability of lung cancer cells transfected with two effective siRNAs for $M M S 22 L$ (si-\#1 and si-\#2) were reduced in correlation with the reduction of MMS22L expression level, implying essential role of MMS22L in the growth of cancer cells (Fig. 2b and c). Since our original gene expression profile database also revealed its high level of expression in clinical cervical cancers, we also knocked down the expression of $M M S 22 L$ by siRNAs in a cervical cancer cell line, HeLa, and observed the growth suppressive effect by siRNAs for $M M S 22 L$.

To further examine the effect of MMS22L overexpression on the growth of mammalian cells, we transiently transfected plasmid designed to express Flag-tagged MMS22L (pCAGGSn-3xFlag-MMS22L) or mock plasmid into COS-7 or HEK293 cells that expressed endogenous MMS22L at very low level. The significant growth promoting effect was observed in the cells transfected with the MMS22L expressing vector compared to those transfected with the mock vector (Fig. 2d).

NFKBIL2 controls the nuclear localization and stability of MMS22L protein. To investigate the biological function of
MMS22L protein, we screened MMS22L-interacting proteins in lung cancer cells using mass spectrometric analysis and identified the interaction between MMS22L and NFKBIL2 [nuclear factor of kappa (NFKB) light polypeptide gene enhancer in B-cells inhibitor-like 2]. Previous reports independently suggested the roles of MMS22L-NFKBIL2 interaction in genomic stability and DNA replication in immortalized cell lines (46-49), however, no study has indicated critical roles of activation of MMS22L and NFKBIL2 in clinical cancers and investigated their functional importance in carcinogenesis. Western blot analysis using cell lines derived from lung cancers and antibodies to MMS22L and NFKBIL2 revealed the co-expression of these two proteins (data not shown), suggesting some functional roles of their interaction in human carcinogenesis. Therefore, we next performed immunofluorescence analysis to determine the subcellular localization of endogenous MMS22L and NFKBIL2 in various cancer cell lines including A549, LC319 and HeLa cells, and found that endogenous MMS22L and NFKBIL2 proteins were mainly co-localized in the nucleus (representative data of HeLa cells was shown in Fig. 3a). To examine the importance of MMS22L-NFKBIL2 interaction in cellar localization of these proteins, we transiently co-expressed exogenous MMS22L and NFKBIL2 proteins using mammalian COS-7 or NIH3T3 cells that expressed these two proteins at very low levels. We found that exogenous MMS22L was mainly located in the cytoplasm and weakly in the nucleus of the cells in which exogenous NFKBIL2 protein was not introduced. However, the nuclear staining of MMS22L was significantly enhanced when both exogenous MMS22L and NFKBIL2 proteins were introduced in the cells (Fig. 3b). On the other hand, exogenous NFKBIL2 was mainly present in the nucleus of cells regardless to the presence or absence of exogenous MMS22L. In addition, we performed western blot analysis using fractionated cytoplasmic and nuclear lysates from COS-7 cells that were introduced exogenous MMS22L and NFKBIL2 proteins. When we transfected both MMS22L-Flag and NFKBIL2-HA expressing vectors, the amounts of nuclear MMS22L was significantly increased, compared with the cells transfected with MMS22L alone (Fig. 3c). Furthermore, we found that knockdown of endogenous MMS22L with siRNA for MMS22L (si-MMS22L) reduced NFKBIL2 protein level in lung cancer LC319 cells and that reduction of NFKBIL2 with si-NFKBIL2 reduced MMS22L levels and significantly suppressed cancer cell growth (Fig. 3d; data not shown). These data suggest that the expression of NFKBIL2 is likely to promote nuclear localization and stability of MMS22L protein, and a complex including these two proteins could coordinately play pivotal roles in cell growth and/or survival.

C-terminal portion of NFKIL2 protein is crucial for binding to MMS22L protein. To examine whether the MMS22L-NFKBIL2 protein complex may play important roles in carcinogenesis, we subsequently constructed various plasmids expressing partial MMS22L proteins with Flag tag or partial NFKBIL2 proteins with HA tag, and transfected them into COS-7 cells (data not shown). Immunoprecipitation and western blotting assays using antibodies to Flag- or HA-tags revealed that an $\mathrm{N}$-terminal portion of MMS22L protein (M1; codon 1-414) could bind to a C-terminal region of NFKBIL2 (N3; codon 
a

Clinical lung cancers

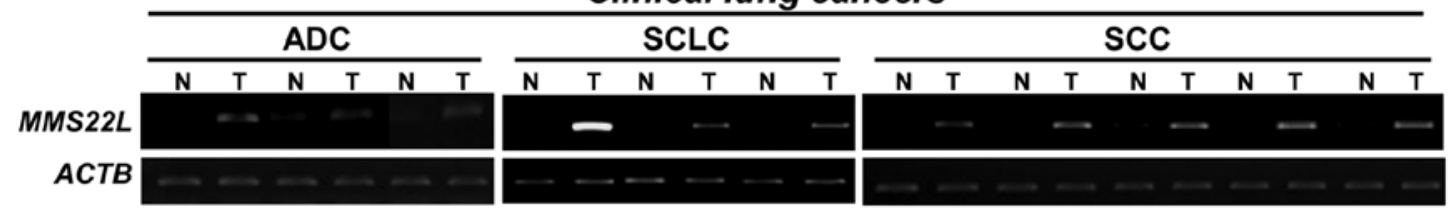

Clinical esophageal cancers

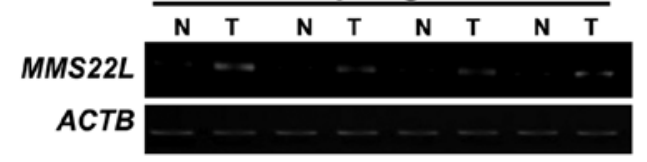

b

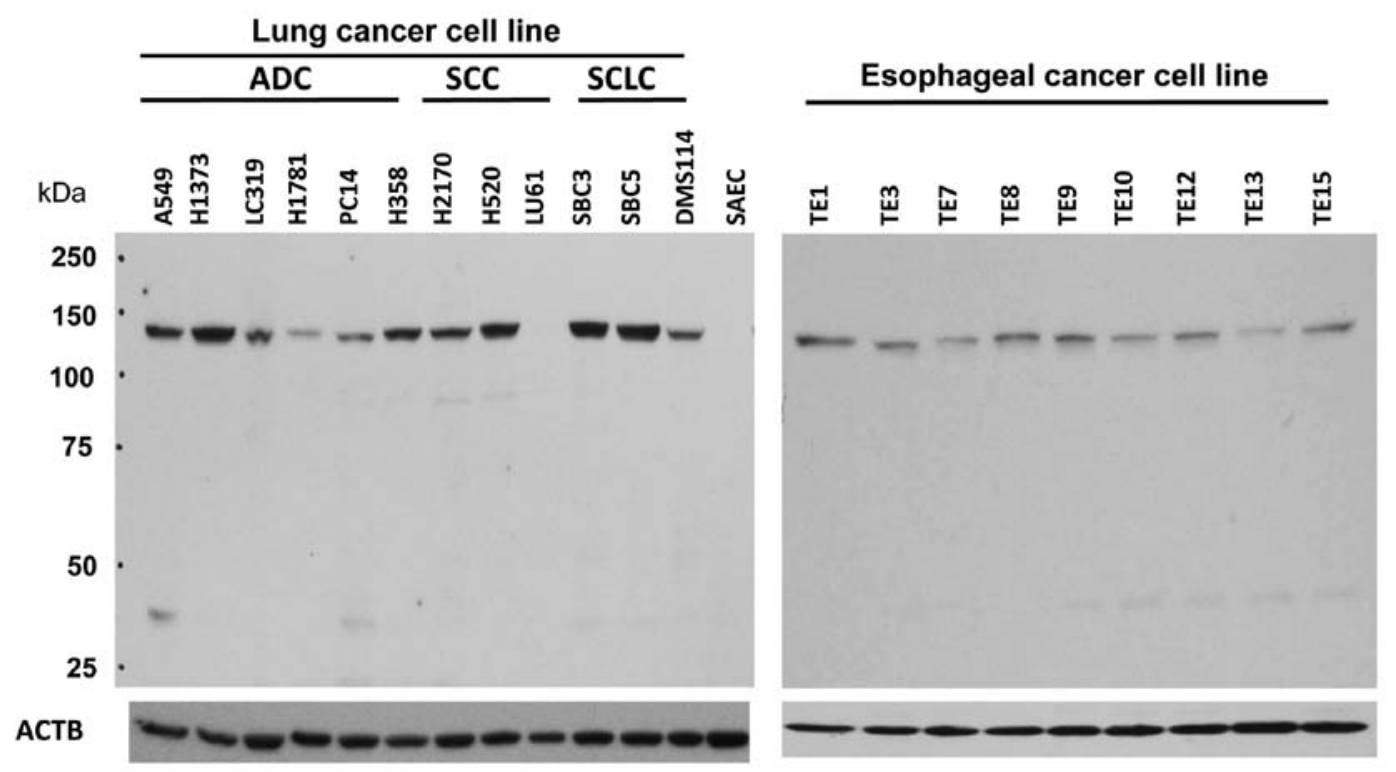

C

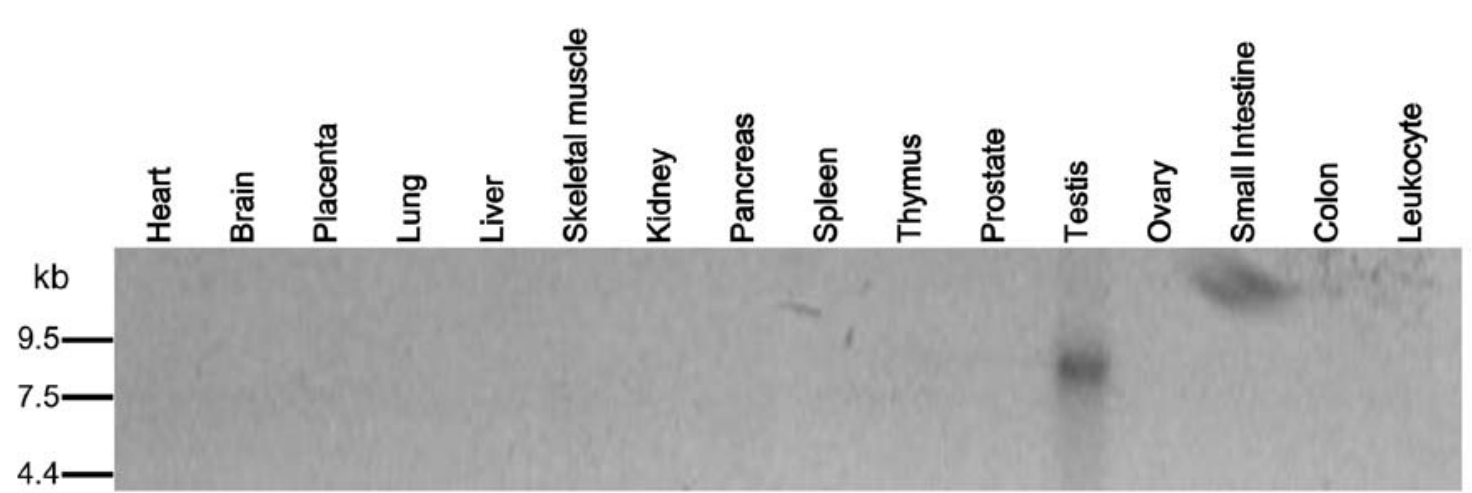

Figure 1. Expression of MMS22L in lung and esophageal cancers and normal tissues. (a) Expression of $M M S 22 L$ gene in (T) lung and esophageal cancer tissues and (N) adjacent normal lung and esophagus tissues, detected by semiquantitative RT-PCR. (b) Expression of MMS22L protein in lung and esophageal cancer cell lines, detected by western blot analysis. (c) Northern blot analysis of the $M M S 22 L$ transcript in 16 normal human tissues.

823-1244) (Fig. 4a). Because immunocytochemical analysis revealed that nuclear localization of MMS22L protein appeared to require the presence of NFKIL2 protein in the nucleus (Fig. 3b), we subsequently investigated which part of NFKBIL2 protein is essential for subcellular localization of MMS22L protein in cultured cells. Plasmids expressing partial proteins of NFKBIL2 were co-transfected with full-length MMS22L expression vector into COS-7 cells. Interestingly, N-terminal
(N1; codon 1-450) and central part (N2; codon 403-836) of NFKBIL2 proteins could be localized in the nucleus, while aggregated MMS22L protein was mainly located in the cytoplasm of the same cells (Fig. 4b). It is concordant with the data that these two partial proteins (N1 and N2) could not bind to MMS22L protein as indicated by immunoprecipitation analyses. In contrast, MMS22L protein and C-terminal part of NFKBIL2 protein (N3; codon 823-1244) that could bind to 
a

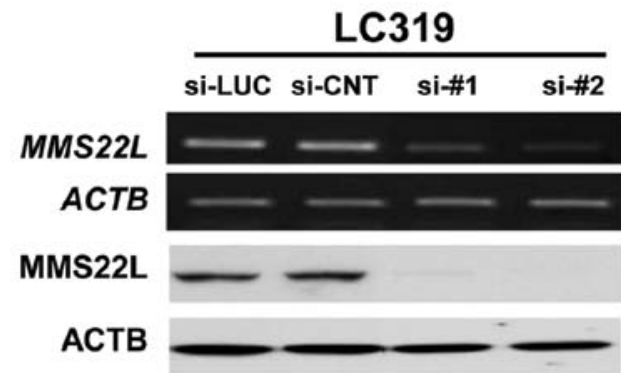

b

C

d

cos-7

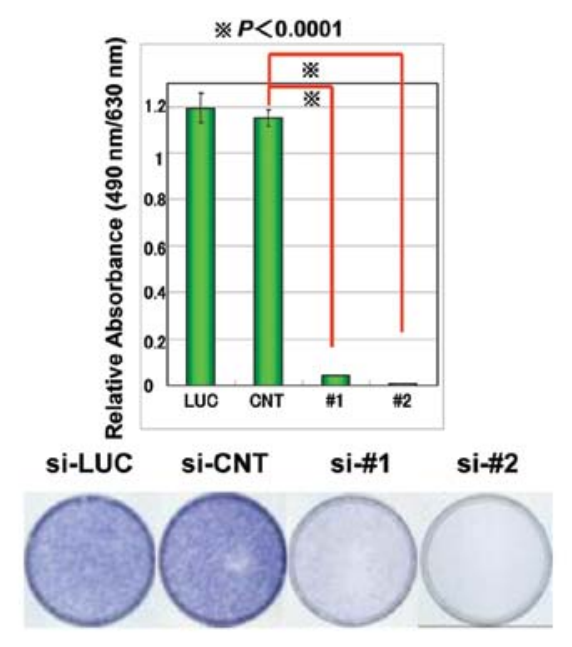

MMS22L

ACTB

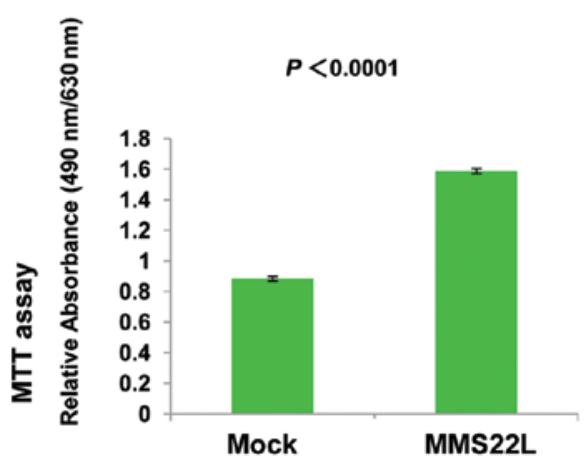

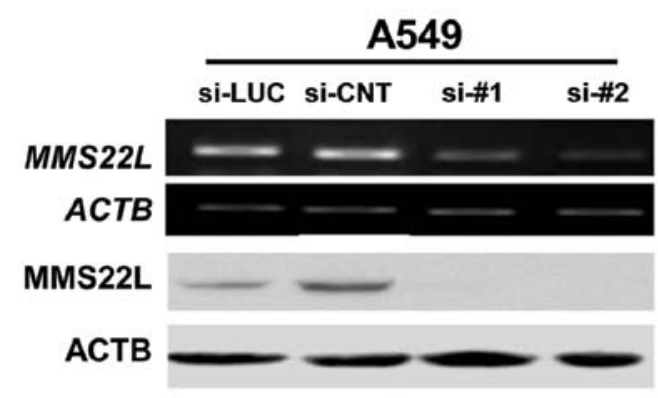
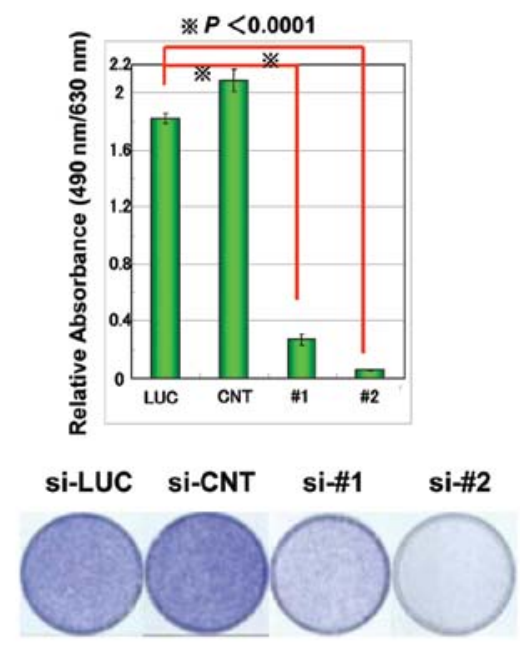

HEK 293
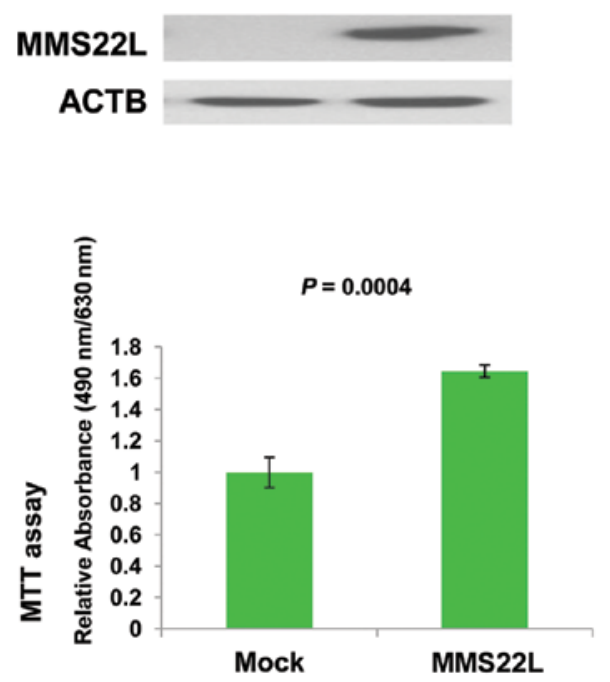

Figure 2. Growth effect of MMS22L. (a) Knockdown of MMS22L expression in lung cancer cell lines, LC319 and A549 by specific siRNA oligonucleotides for MMS22L (si-\#1 and si-\#2) or control siRNAs (si-LUC and si-CNT), confirmed by semiquantitative RT-PCR and western blot analyses. (b) Viability of A549 and LC319 cells evaluated by MTT assay in response to the siRNAs. All assays were performed in triplicate wells at three independent times. (c) Colony formation assays using LC319 and A549 cells transfected with the siRNAs. (d) Enhanced growth promoting activity of COS-7 and HEK293 cells transfected with mock or MMS22L expressing vectors as quantified by MTT assay at 7 days after transfection.

MMS22L protein were mainly localized in the cytoplasm of the cells (Fig. 4b). The data indicate that $\mathrm{N}$-terminal (N1; codon 1-450) and central (N2; codon 403-836) parts of NFKBIL2 are more important for nuclear localization of NFKBIL2, while its C-terminal part (N3; codon 823-1244) is essential for binding to MMS22L.
Dominant negative growth suppressive effect of partial NFKBIL2 protein including MMS22L-binding site. According to the data above, we hypothesized that if nuclear localization of MMS22L protein is important for cancer cells growth, reduction of MMS22L protein in the nucleus by inhibiting the interaction between MMS22L and NFKBIL2 could suppress the cancer cell 
a

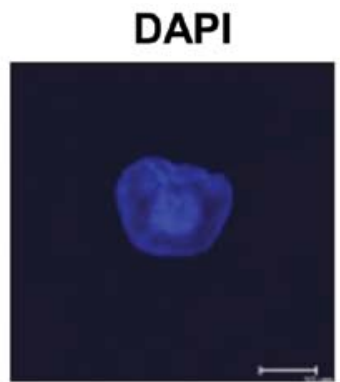

b
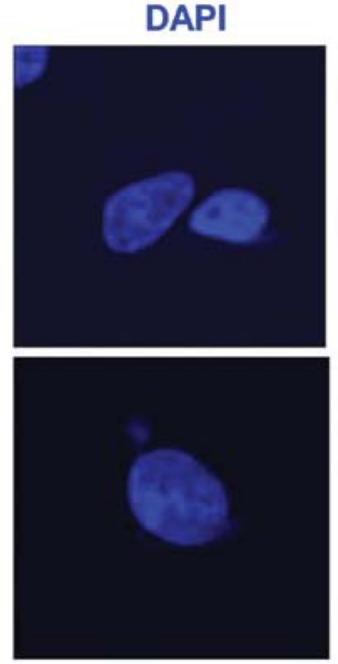

C
MMS22L

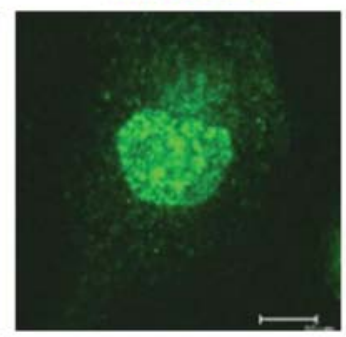

MMS22L
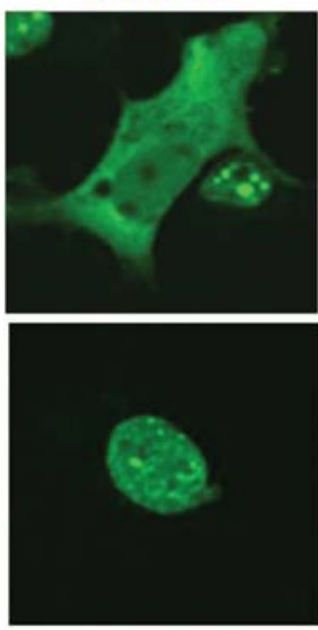

NFKBIL2

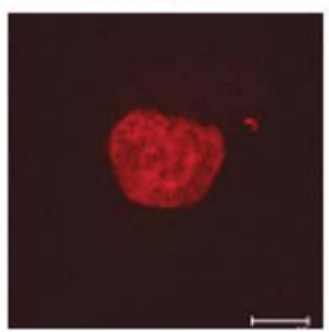

NFKBIL2
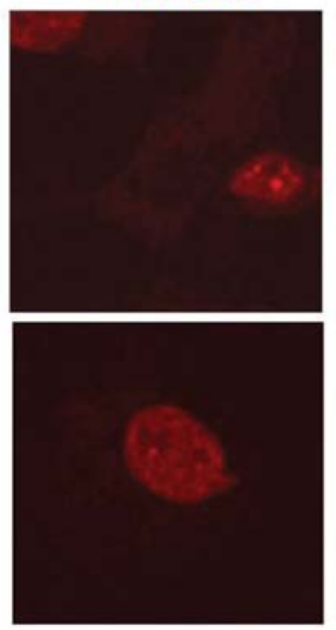

MERGE

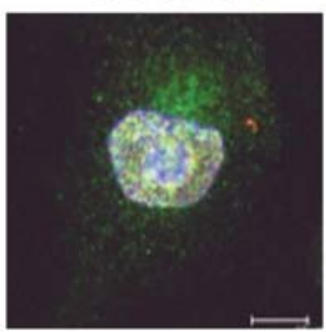

MERGE
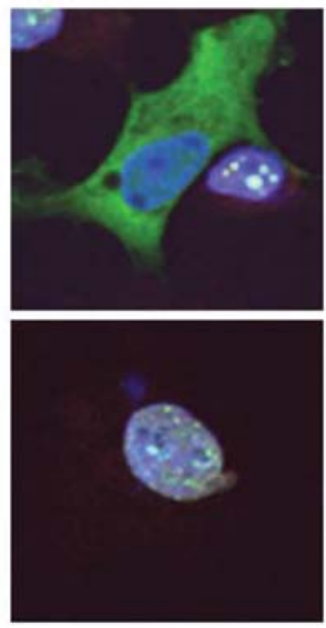

\section{MMS22L-Flag \\ HA-NFKBIL2 \\ Mock-Flag \\ HA-Mock}

$$
\begin{aligned}
& -+-+\overline{-}+\overline{-}+\bar{t} \\
& --++-1+-1+ \\
& +--+-+-+-+ \\
& ++
\end{aligned}
$$

MMS22L-Flag

NFKBIL2-HA

Hsp90

Lamin B

\section{ACTB}

d
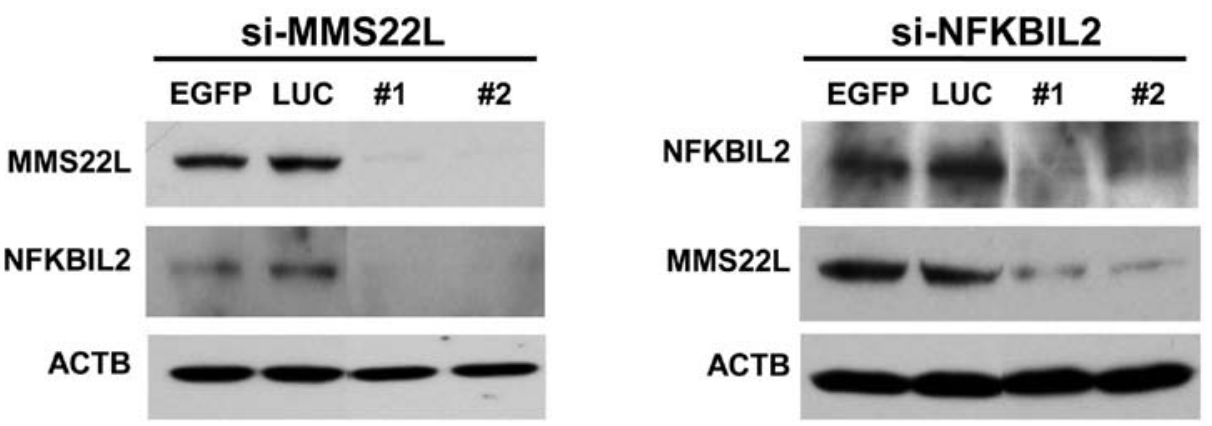

Figure 3. Nuclear localization and stability of MMS22L and its interacting protein of NFKBIL2 in cultured cells. (a) Subcellular localization of endogenous MMS22L and NFKBIL2 proteins in HeLa cells. The cells were stained with a rabbit polyclonal anti-MMS22L antibody (green); mouse polyclonal anti-NFKBIL2 (red) and with DAPI (blue). (b) Subcellular localization of MMS22L in the presence or absence of NFKBIL2 protein. COS-7 cells were co-transfected with Flag-tagged MMS22L- and HA-tagged NFKBIL2-expression vectors. MMS22L protein was stained with anti-Flag M2 antibody (green); NFKBIL2 with anti-HA (red) and nucleus with DAPI (blue). (c) Western blot analysis using cytoplasmic and nuclear fraction of COS-7 cells which were co-transfected with MMS22L- and NFKBIL2- expression vectors. MMS22L and NFKBIL2 proteins were detected using anti-Fag-M2 antibody and/or anti-HA (3F10) antibody. (d) Knockdown of MMS22L or NFKBIL2 protein expression with si-MMS22L or si-NFKBIL2 oligonucleotides. The expression of endogenous MMS22L and NFKBIL2 proteins were detected by western blot analysis using anti-MMS22L antibody and anti-NFKBIL2 antibody. 
a

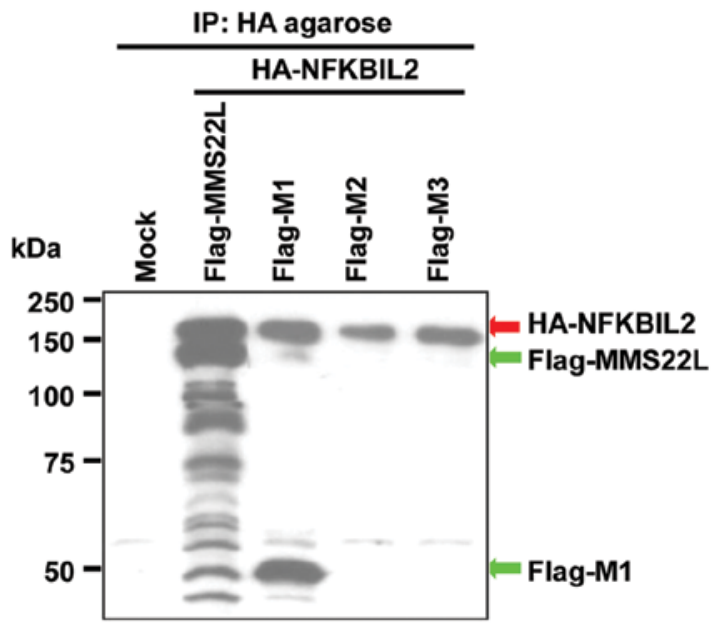

IP: Flag agasrose

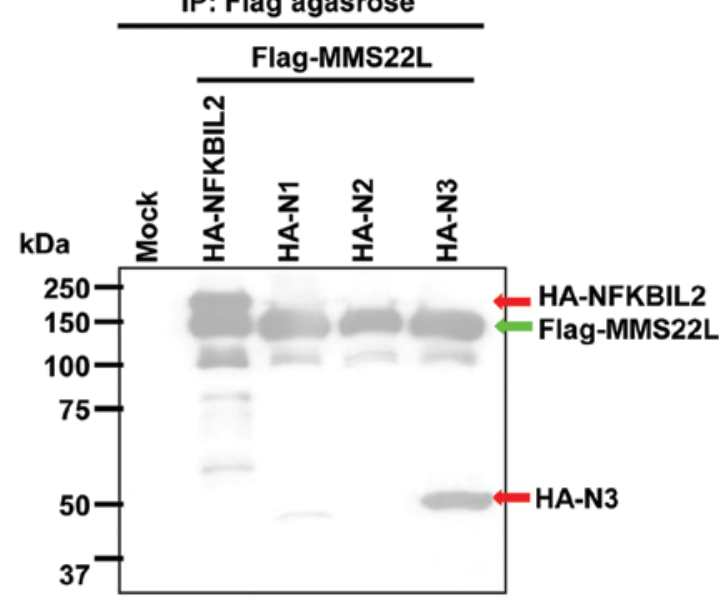

NFKBIL2

MERGE

b
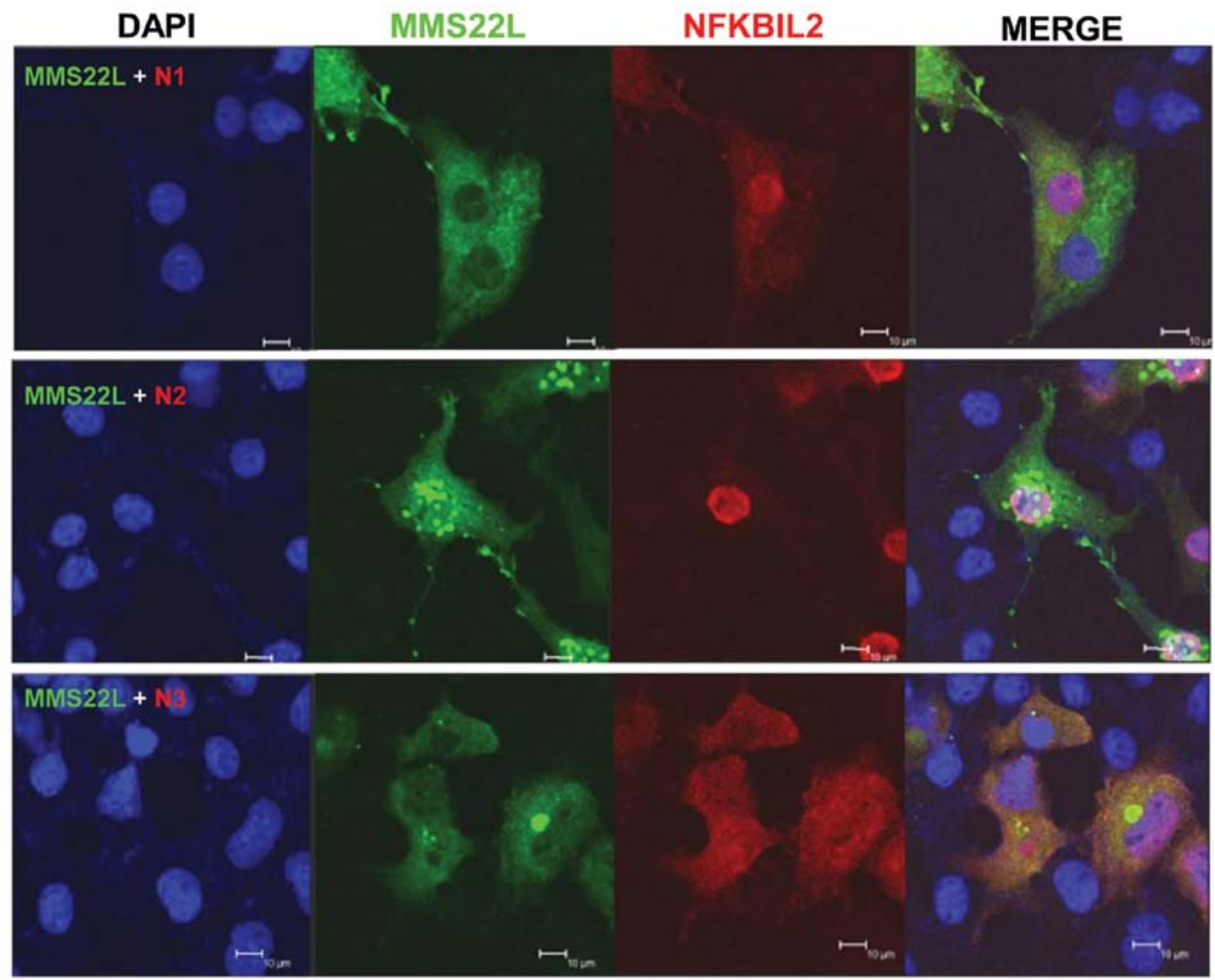

Figure 4. C-terminal portion of NFKIL2 protein is crucial for binding to MMS22L protein. (a) Binding between MMS22L and NFKBIL2 proteins detected using Flag- or HA-agarose and COS-7 cells co-transfected with full-length/partial MMS22L-Flag and full-length/partial NFKBIL2-HA proteins. (b) Subcellular localization of full-length MMS22L and three partial NFKBIL2 proteins. COS-7 cells were co-transfected with full-length MMS22L and partial NFKBIL2-expression vectors (N1-N3). MMS22L protein was stained with anti-Flag M2 antibody (green); NFKBIL2 with anti-HA antibody (red) and nucleus with DAPI (blue).

growth. To examine whether exogenous expression of partial N3 protein can inhibit the MMS22L-NFKBIL2 interaction and cell growth, we co-transfected full-length MMS22L and either of full-/partial-length NFKBIL2 expressing vectors (N1, N2 or N3) into HEK293 cells, and found that the amount of exogenous full-length NFKBIL2 protein that binds to exogenous MMS22L was significantly decreased after introduction of the partial $\mathrm{N} 3$ protein, as demonstrated by immunoprecipitation assays, while it was not changed in the cells transfected with $\mathrm{N} 1$ or N2 vectors (Fig. 5a). To investigate the functional significance of the interaction between MMS22L and NFKBIL2 for growth of cancer cells, we transfected either of vectors expressing partial NFKBIL2 proteins or mock vectors into two cancer cell lines, HeLa and LC319, which highly expressed both endogenous MMS22L and NFKBIL2 proteins and lung fibroblast CCDlu-19 cells in which MMS22L expression was hardly detectable. Expectedly, exogenous expression of the C-terminal portion of NFKIL2 protein (N3) reduced the levels of MMS22L protein in the nucleus and inhibited the growth of HeLa and LC319 cells as measured by MTT assay, while it did not affect the growth of MMS22L-negative CCDLu-19 cells (Figs. 5b-d). Our findings imply that inhibition of the interaction between the MMS22L 
a
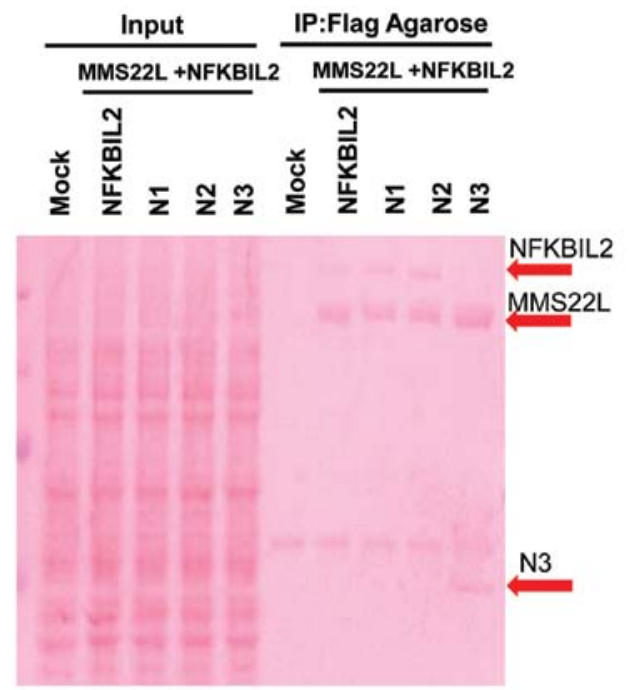

Ponsauce S stain

b

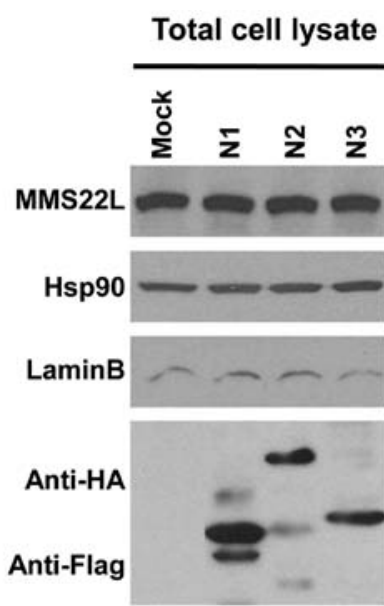

c

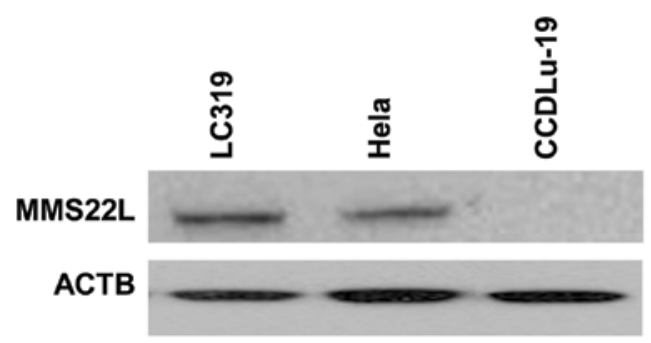

d
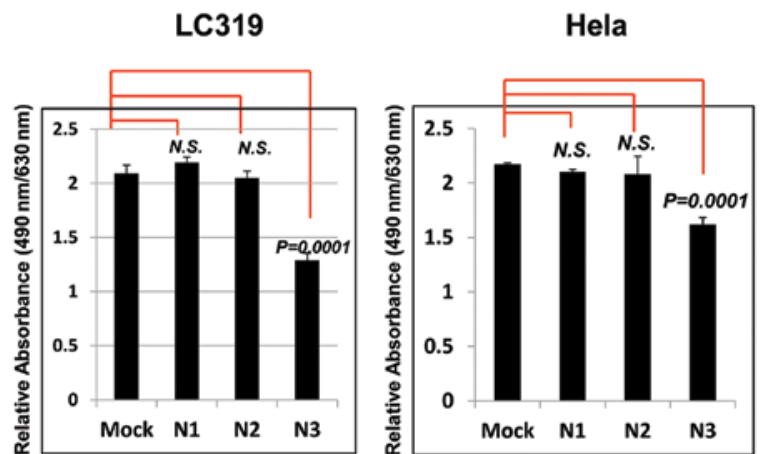

Input

IP:Flag Agarose

MMS22L +NFKBIL2 MMS22L +NFKBIL2
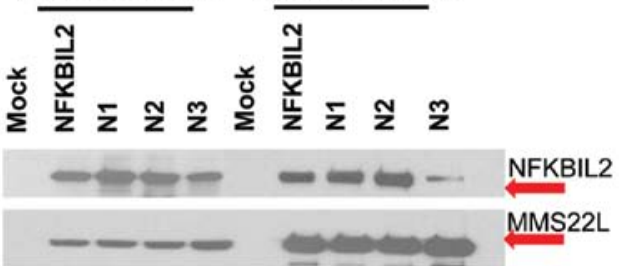

АСтB

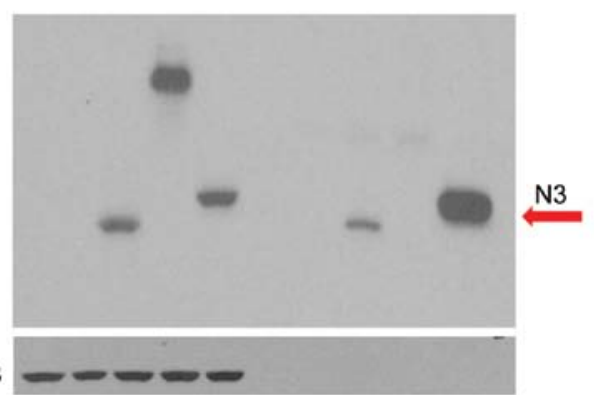

Cytoplasm

Nucleus
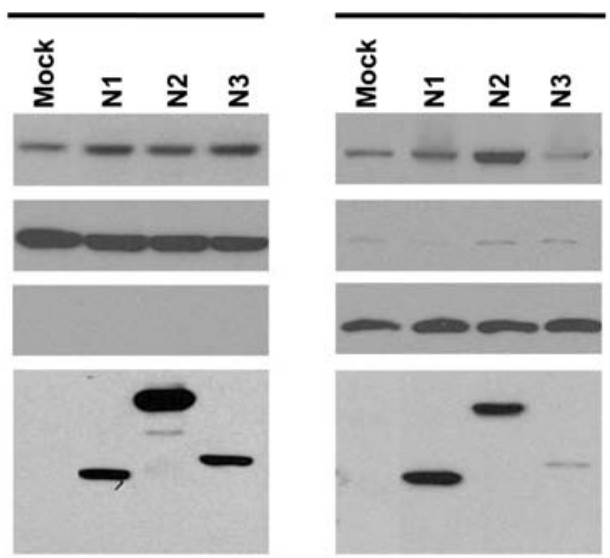

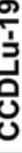

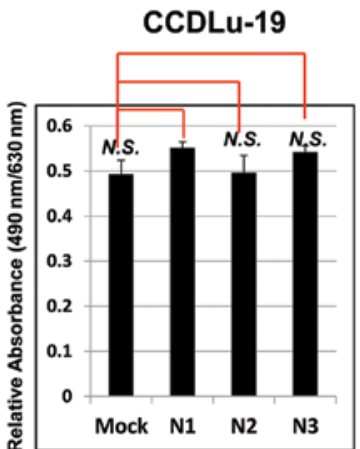

Figure 5. Dominant negative growth suppressive effect of partial NFKBIL2 protein on cancer cells. (a) HEK293 cells which were co-transfected with MMS22Land full-length/three partial NFKBIL2-expression vectors (N1, N2, and N3). Immunoprecipitation assays were performed using Flag-M2 agarose. (b) Western blot analysis using cytoplasmic and nuclear fractionation of the lysates from HeLa cells which were transfected with partial NFKBIL2-expressing vectors. Endogenous MMS22L was detected using anti-MMS22L antibody. Partial NFKBIL2 proteins were detected with anti-rat HA (3F10) antibody. (c) The expression of MMS22L protein in HeLa, LC319 and CCDlu-19 cell lines. (d) MTT assay using MMS22L-positive HeLa and LC319 cells, and MMS22L-negative CCDlu-19 cells, which were transfected with mock plasmids or either of three partial NFKBIL2-expression vectors (N1, N2 and N3), as quantified by MTT assay at 7 days after transfection. 
a

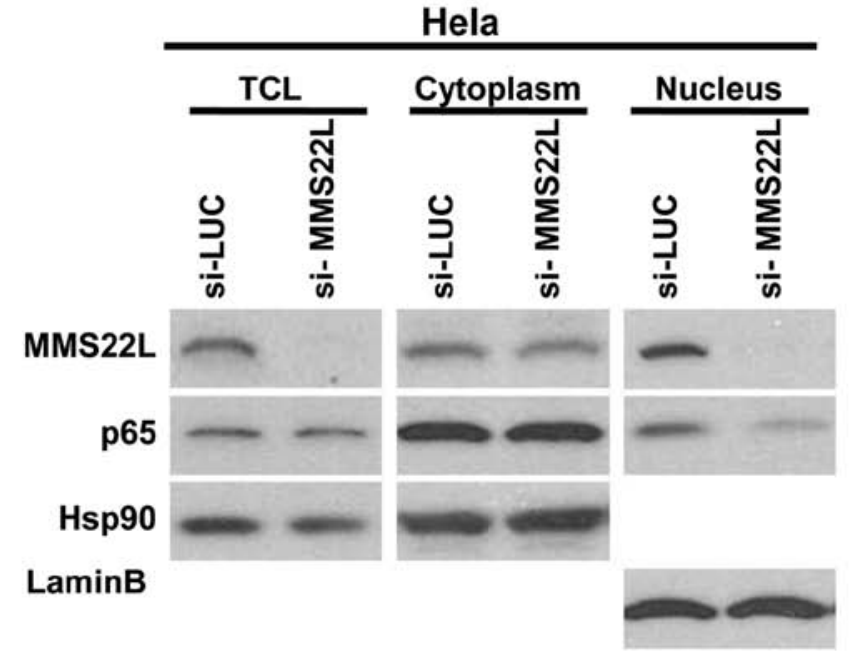

LC319

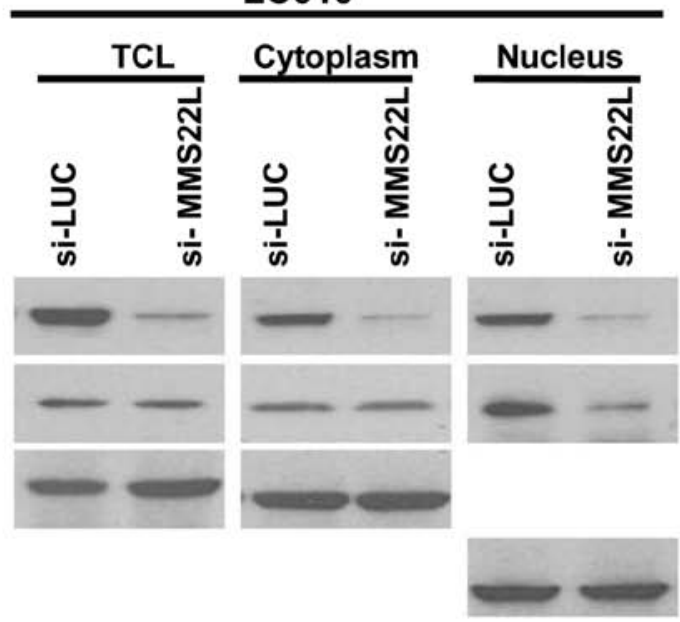

b

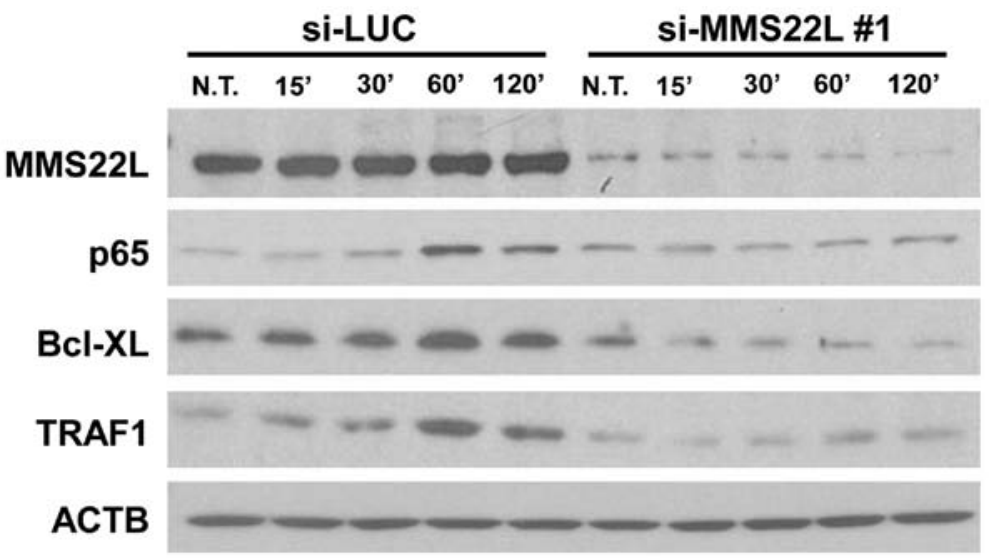

C
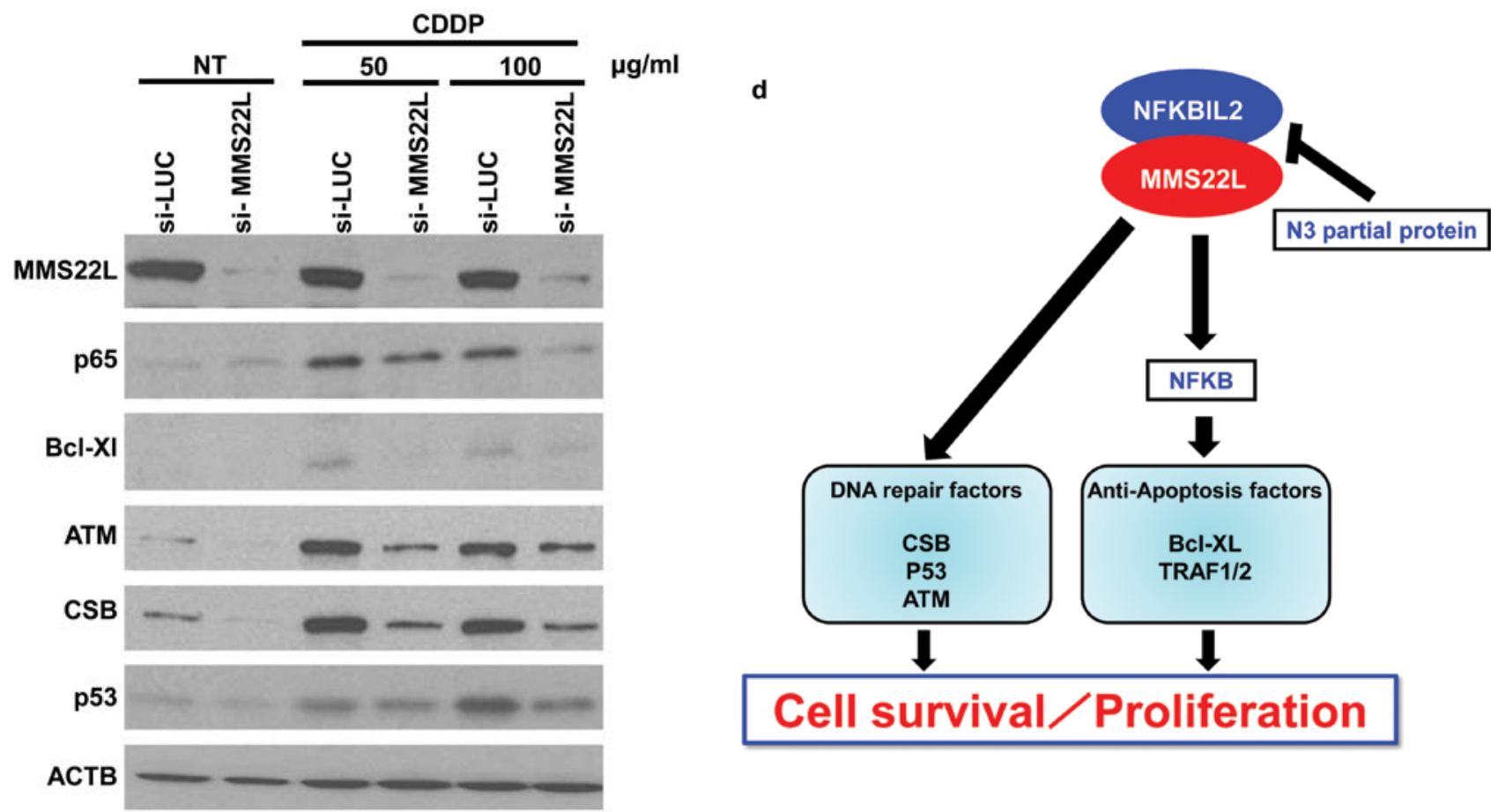

Figure 6. Involvement of MMS22L as an upstream molecule of NFKB pathway. (a) Western blot analysis using antibodies to endogenous MMS22L and RelA/p65, and HeLa and LC319 cells transfected with siRNA oligonucleotides for MMS22L (si-MMS22L) or control siRNA (si-LUC). These cell lines were stimulated with $50 \mathrm{ng} / \mathrm{ml} \mathrm{TNF}-\alpha$ for $15 \mathrm{~min}$. The nuclear and cytoplasmic fraction was isolated using NE-PER ${ }^{\mathrm{TM}}$ Nuclear and Cytoplasmic Extraction Reagents kit (Thermo). (b) Western blot analysis using antibodies to endogenous MMS22L, RelA/p65, Bcl-XL and TRAF1 and HeLa cells transfected with si-MMS22L or si-LUC. These cell lines were treated with $50 \mathrm{ng} / \mathrm{ml}$ TNF- $\alpha$ for $15,30,60$ or 120 min. N.T. indicates no treatment with TNF- $\alpha$. (c) Western blot analysis using antibodies to endogenous MMS22L, RelA/p65, Bcl-XL, ATM, CSB, p53 and HeLa cells which were transfected with si-MMS22L or si-LUC oligonucleotides, and were subsequently treated with cisplatin (CDDP; 50 or $100 \mu \mathrm{g} / \mathrm{ml}$ ). (d) Schematic summary of MMS22L pathway. 
and NFKBIL2 protein can suppress the nuclear localization of MMS22L protein, and resulted in the reduction of cancer growth, and that inhibition of the interaction in cancer cells by small molecules might be a potential therapeutic strategy for new cancer treatment.

$M M S 22 L$ protein acts as an upstream molecule of NFKB pathway. Since NFKIL2 protein was indicated to be involved in the NFKB pathway that plays an essential role in the promotion of cell proliferation and anti-apoptosis (42-45), we examined the expression of NFKB p65/RelA protein in HeLa cells in which both exogenous MMS22L and NFKIL2 were introduced, and found that the level of endogenous p65/RelA protein was elevated compared with those of cells introduced NFKIL2 alone (data not shown). The result suggests that the expression of MMS22L-NFKBIL2 complex may positively regulate the NFKB pathway. Subsequently, we attempted to examine the effect of endogenous MMS22L expression on the NFKB pathway molecules using cytoplasmic and nuclear fraction of HeLa and LC319 cells that were treated with TNF- $\alpha$. We first confirmed that the level of RelA/p65 was increased in the nucleus of the cells by TNF- $\alpha$ stimulation (data not shown), but that of endogenous RelA/p65 protein was decreased in these cells transfected with siRNA for MMS22L (si-MMS22L) after TNF- $\alpha$ treatment, compared to cells with control siRNA (si-LUC) (Fig. 6a). We then examined the relationship between MMS22L protein and downstream molecules of RelA/p65 such as Bcl-XL and TRAF1/2 that were the anti-apoptosis factors. When we treated the si-LUC-transfected HeLa cells with TNF- $\alpha, \mathrm{Bcl}-\mathrm{XL}$ and TRAF1 were increased in accordance with the elevation of RelA/p65 (Fig. 6b). However, the elevation of p65, Bcl-XL and TRAF1 were not detected in the TNF- $\alpha$-stimulated cells transfected with si-MMS22L. The expression level of MMS22L protein showed good correlation with those of $\mathrm{p} 65, \mathrm{Bcl}-\mathrm{XL}$ and TRAF1 proteins in lung cancer cell lines (data not shown).

To further examine the effect of MMS22L expression on apoptosis pathway in cancer cells, we cultured cancer cells that were transfected with si-MMS22L under DNA damage condition using DNA-damaging agents (cisplatin/CDDP or 5-fluorouracil/5-FU). After knockdown of $M M S 22 L$ expression with si-MMS22L in HeLa cells, we treated the cells with CDDP $(50 \mu \mathrm{g} / \mathrm{ml})$ or 5 -FU $(50 \mu \mathrm{g} / \mathrm{ml})$ for $48 \mathrm{~h}$ and harvested the cells for flow cytometric analysis. The sub G1 population of the cells which were transfected with si-MMS22L was significantly increased compared with those with control siRNA (si-LUC) under DNA damage condition (data not shown). When we exposed the cells that were transfected with si-MMS22L or si-LUC with $20 \mathrm{~J}$ of ultraviolet for $48 \mathrm{~h}$, the similar results were observed (data not shown). Western blot analysis using the HeLa cells which were transfected with si-MMS22L or si-LUC, and subsequently treated with CDDP as mentioned above revealed that induction of DNA repair molecules such as ATM, CSB and p53 as well as RelA/p65 and its downstream anti-apoptosis factor Bcl-XL were significantly suppressed in the cells transfected with si-MMS22L compared with those transfected with si-LUC (Fig. 6c). The data suggest that MMS22L can function as an upstream molecule of these anti-apoptosis factors and also affect the induction of some DNA repair pathway molecules (Fig. 6d).

\section{Discussion}

Despite the recent development of surgical techniques combined with various treatment modalities such as radiotherapy and chemotherapy, clinical outcome of lung and esophageal cancer patients still remains poor. Therefore, development of new types of anticancer drugs is eagerly awaited. To identify novel target molecules for drug development, we combined genome-wide expression profile analysis of genes that were overexpressed in lung and esophageal cancer cells with high-throughput screening of loss-of-function effects by means of the RNAi technique and tumor tissue microarray analysis (5-41). Through this systematic approach we found $M M S 22 L$ to be upregulated frequently in clinical lung and esophageal cancer samples, and showed that this gene product plays an indispensable role in the growth and/or survival of cancer cells.

We demonstrated that MMS22L is a putative oncogene and that its nuclear localization and stabilization was enhanced by binding to NFKBIL2. In addition, we revealed that introduction of the C-terminal portion of NFKBIL2 protein into cancer cells could dominant-negatively inhibit the nuclear localization of MMS22L possibly by blocking the MMS22L-NFKBIL2 interaction, and resulted in the suppression of cancer cell growth/ survival. Furthermore, transfection of siRNAs against MMS22L or NFKBIL2 into cancer cells suppressed their expression and the cell growth. Therefore, inhibition of the MMS22L-NFKBIL2 interaction or suppressing MMS22L protein function can be an effective approach for development of novel cancer therapy.

To date, NFKB transcription factors are known to be the key regulators of immune, inflammatory and acute phase responses, and to be involved in the control of cell proliferation and apoptosis (42-45). Activation of NFKB activity and consequent induction of its downstream genes lead to the oncogenesis in mammalian cells. MMS22L protein appeared to act as an upstream molecule of RelA/p65 and be indispensable for induction of anti-apoptosis factors, Bcl-XL or TRAF1. Further studies on the regulation and function of MMS22L protein will contribute to the understanding of molecular mechanism of carcinogenesis through the activation of MMS22L and NFKB pathway.

In cancer chemotherapy, many kinds of DNA damaging agents are being used. The most common approach for targeting the cell cycle is to exploit the effect of DNA-damaging chemotherapeutic agents like 5-FU or CDDP, whose effects are mediated through diverse intracellular targets inducing apoptosis in various cancer cells (50). However, the toxicity of DNA-damaging drugs can be diminished by the activities of several DNA repair pathways as well as anti-apoptotic factors. Therefore, inhibitors of specific DNA repair and/or anti-apoptotic pathways might be promising therapeutic strategy for novel cancer treatments which can improve the efficacy of DNA damage-based cancer therapy (50). Our data suggested the involvement of MMS22L in cellular response to DNA damaging agents. In fact, knockdown of MMS22L expression also enhanced the apoptosis of cancer cells that were exposed to DNA-damaging agents including 5-FU and CDDP probably due to inhibition of induction of DNA repair molecules such as ATM, CSB and p53 as well as RelA/p65 and its downstream anti-apoptosis factor Bcl-XL. The combined data of our experiments suggest that MMS22L might function as an upstream molecule of these anti-apoptosis factors and DNA-repair molecules and that targeting MMS22L could 
have a significant advantage in avoiding the resistance of cancer cells to anticancer treatments, although the detailed function of MMS22L in drug response of the cells and in carcinogenesis remains to be elucidated.

In summary, our data indicate that MMS22L is involved in NFKB pathway in cancer cells through its interaction with NFKBIL2 and that it might be a promising candidate target for developing highly specific anticancer drugs with minimal risk of adverse effects.

\section{Acknowledgements}

This study was supported in part by Grant-in-Aid for Scientific Research (B) and Grant-in-Aid for Scientific Research on Innovative Areas from The Japan Society for the Promotion of Science to Y.D. Y.D. is a member of Shiga Cancer Treatment Project supported by Shiga Prefecture (Japan).

\section{References}

1. Jemal A, Siegel R, Ward E, Hao Y, Xu J and Thun MJ: Cancer statistics, 2009. CA Cancer J Clin 59: 225-249, 2009.

2. Shimada H, Nabeya Y, Okazumi S, et al: Prediction of survival with squamous cell carcinoma antigen in patients with resectable esophageal squamous cell carcinoma. Surgery 133: 486-494, 2003.

3. Berwick M and Schantz S: Chemoprevention of aerodigestive cancer. Cancer Metastasis Rev 16: 329-347, 1997.

4. Daigo Y and Nakamura Y: From cancer genomics to thoracic oncology: discovery of new biomarkers and therapeutic targets for lung and esophageal carcinoma. Gen Thorac Cardiovasc Surg 56: 43-53, 2008

5. Kikuchi T, Daigo Y, Katagiri T, et al: Expression profiles of non-small cell lung cancers on cDNA microarrays: identification of genes for prediction of lymph-node metastasis and sensitivity to anti-cancer drugs. Oncogene 22: 2192-205, 2003.

6. Kakiuchi S, Daigo Y, Tsunoda T, Yano S, Sone S and Nakamura Y: Genome-wide analysis of organ-preferential metastasis of human small cell lung cancer in mice. Mol Cancer Res 1: 485-499, 2003.

7. Kakiuchi S, Daigo Y, Ishikawa N, et al: Prediction of sensitivity of advanced non-small cell lung cancers to gefitinib (Iressa, ZD1839). Hum Mol Genet 13: 3029-43, 2004.

8. Kikuchi T, Daigo Y, Ishikawa N, et al: Expression profiles of metastatic brain tumor from lung adenocarcinomas on cDNA microarray. Int J Oncol 28: 799-805, 2006.

9. Taniwaki M, Daigo Y, Ishikawa N, et al: Gene expression profiles of small-cell lung cancers: molecular signatures of lung cancer. Int J Oncol 29: 567-575, 2006.

10. Yamabuki T, Daigo Y, Kato T, et al: Genome-wide gene expression profile analysis of esophageal squamous cell carcinomas. Int J Oncol 28: 1375-1384, 2006.

11. Suzuki C, Daigo Y, Kikuchi T, Katagiri T and Nakamura Y: Identification of COX17 as a therapeutic target for non-small cell lung cancer. Cancer Res 63: 7038-7041, 2003.

12. Kato T, Daigo Y, Hayama S, et al: A novel human tRNA-dihydrouridine synthase involved in pulmonary carcinogenesis. Cancer Res 65: 5638-5646, 2005.

13. Furukawa C, Daigo Y, Ishikawa N, et al: Plakophilin 3 oncogene as prognostic marker and therapeutic target for lung cancer. Cancer Res 65: 7102-7110, 2005.

14. Suzuki C, Daigo Y, Ishikawa N, et al: ANLN plays a critical role in human lung carcinogenesis through the activation of RHOA and by involvement in the phosphoinositide 3-kinase/AKT pathway. Cancer Res 65: 11314-11325, 2005.

15. Ishikawa N, Daigo Y, Takano A, et al: Characterization of SEZ6L2 cell-surface protein as a novel prognostic marker for lung cancer. Cancer Sci 97: 737-745, 2006.

16. Takahashi K, Furukawa C, Takano A, et al: The neuromedin $\mathrm{u}$-growth hormone secretagogue receptor $1 \mathrm{~b} /$ neurotensin receptor 1 oncogenic signaling pathway as a therapeutic target for lung cancer. Cancer Res 66: 9408-9419, 2006.
17. Hayama S, Daigo Y,Kato T, et al: Activation of CDCA1-KNTC2, members of centromere protein complex, involved in pulmonary carcinogenesis. Cancer Res 66: 10339-10348, 2006.

18. Kato T, Hayama S, Yamabuki T, et al: Increased expression of IGF-II mRNA-binding protein 1 is associated with the tumor progression in patients with lung cancer. Clin Cancer Res 13: 434-442, 2007.

19. Suzuki C, Takahashi K, Hayama S, et al: Identification of Myc-associated protein with $\mathrm{JmjC}$ domain as a novel therapeutic target oncogene for lung cancer. Mol Cancer Ther 6: 542-551, 2007.

20. Hayama S, Daigo Y, Yamabuki T, et al: Phosphorylation and activation of cell division cycle associated 8 by aurora kinase $B$ plays a significant role in human lung carcinogenesis. Cancer Res 67: 4113-4122, 2007.

21. Taniwaki M, Takano A, Ishikawa N, et al: Activation of KIF4A as a prognostic biomarker and therapeutic target for lung cancer. Clin Cancer Res 13: 6624-6631, 2007.

22. Mano Y, Takahashi K, Ishikawa N, et al: Fibroblast growth factor receptor 1 oncogene partner as a novel prognostic biomarker and therapeutic target for lung cancer. Cancer Sci 98: 1902-1913, 2007.

23. Kato T, Sato N, Hayama S, et al: Activation of holliday junction recognizing protein involved in the chromosomal stability and immortality of cancer cells. Cancer Res 67: 8544-8553, 2007.

24. Kato T, Sato N, Takano A, et al: Activation of placenta specific transcription factor distal-less homeobox 5 predicts clinical outcome in primary lung cancer patients. Clin Cancer Res 14: 2363-2370, 2008.

25. Dunleavy EM, Roche D, Tagami H, et al: HJURP is a cell-cycle-dependent maintenance and deposition factor of CENP-A at centromeres. Cell 137: 485-497, 2009.

26. Hirata D, Yamabuki T, Miki D, et al: Involvement of epithelial cell transforming sequence- 2 oncoantigen in lung and esophageal cancer progression. Clin Cancer Res 15: 256-266, 2009.

27. Sato N, Koinuma J, Fujita M, et al: Activation of WD repeat and high-mobility group box DNA binding protein 1 in pulmonary and esophageal carcinogenesis. Clin Cancer Res 16: 226-239, 2010.

28. Sato N, Koinuma J, Ito $\mathrm{T}$, et al: Activation of an oncogenic TBC1D7 (TBC1 domain family, member 7 ) protein in pulmonary carcinogenesis. Genes Chromosomes Cancer 49: 353-367, 2010.

29. Nguyen MH,Koinuma J, Ueda K, et al: Phosphorylation and activation of cell division cycle associated 5 by mitogen-activated protein kinase play a crucial role in human lung carcinogenesis. Cancer Res 70: 5337-5347, 2010.

30. Ishikawa N, Daigo Y, Yasui W, et al: ADAM8 as a novel serological and histochemical marker for lung cancer. Clin Cancer Res 10: 8363-8370, 2004

31. Ishikawa N, Daigo Y, Takano A, et al: Increases of amphiregulin and transforming growth factor-alpha in serum as predictors of poor response to gefitinib among patients with advanced non-small cell lung cancers. Cancer Res 65: 9176-9184, 2005.

32. Yamabuki T, Takano A, Hayama S, et al: Dickkopf-1 as a novel serologic and prognostic biomarker for lung and esophageal carcinomas. Cancer Res 67: 2517-2525, 2007.

33. Ishikawa N, Takano A, Yasui W, et al: Cancer-testis antigen lymphocyte antigen 6 complex locus $\mathrm{K}$ is a serologic biomarker and a therapeutic target for lung and esophageal carcinomas. Cancer Res 67: 11601-11611, 2007.

34. Takano A, Ishikawa N, Nishino R, et al: Identification of nectin- 4 oncoprotein as a diagnostic and therapeutic target for lung cancer. Cancer Res 69: 6694-6703, 2009.

35. Sato N, Yamabuki T, Takano A, et al: Wnt inhibitor Dickkopf-1 as a target for passive cancer immunotherapy: Cancer Res 70: 5326-5336, 2010.

36. Suda T, Tsunoda T, Daigo Y, Nakamura $Y$ and Tahara H: Identification of human leukocyte antigen-A24-restricted epitope peptides derived from gene products upregulated in lung and esophageal cancers as novel targets for immunotherapy. Cancer Sci 98: 1803-1808, 2007.

37. Mizukami Y, Kono K, Daigo Y, et al: Detection of novel cancer-testis antigen-specific T-cell responses in TIL, regional lymph nodes, and PBL in patients with esophageal squamous cell carcinoma. Cancer Sci 99: 1448-1454, 2008.

38. Harao M, Hirata S, Irie A, et al: HLA-A2-restricted CTL epitopes of a novel lung cancer-associated cancer testis antigen, cell division cycle associated 1 , can induce tumor-reactive CTL. Int J Cancer 123: 2616-2625, 2008. 
39. Kono K, Mizukami Y, Daigo Y, et al: Vaccination with multiple peptides derived from novel cancer-testis antigens can induce specific T-cell responses and clinical responses in advanced esophageal cancer. Cancer Sci 100: 1502-1509, 2009.

40. Yokomine K, Senju S, Nakatsura T, et al: The forkhead box M1 transcription factor, as a candidate of target for anti-cancer immunotherapy. Int J Cancer 126: 2153-2163, 2010.

41. Tomita Y, Imai K, Senju S, et al: A novel tumor-associated antigen, cell division cycle 45-like can induce cytotoxic T-lymphocytes reactive to tumor cells. Cancer Sci 102: 697-705, 2011.

42. Rayet B and Gelinas C: Aberrant rel/nfkb genes and activity in human cancer. Oncogene 18: 6938-6947, 1999.

43. Tergaonkar V: NFאB pathway: A good signaling paradigm and therapeutic target. Int J Biochem Cell Biol 38: 1647-1653, 2006.

44. Yamamoto $\mathrm{Y}$ and Gaynor RB: Therapeutic potential of inhibition of the NF-kappaB pathway in the treatment of inflammation and cancer. J Clin Invest 107: 135-142, 2001.
45. Kim HJ, Hawke $\mathrm{N}$ and Baldwin AS: NF- $\kappa \mathrm{B}$ and IKK as therapeutic targets in cancer. Cell Death Differ 13: 738-747, 2006.

46. O'Donnell L, PanierS, Wildenhain J, et al: The MMS22L-TONSL complex mediates recovery from replication stress and homologous recombination. Mol Cell 40: 619-631, 2010.

47. Duro E, Lundin C, Ask K, et al: Identification of the MMS22L-TONSL complex that promotes homologous recombination. Mol Cell 40: 632-644, 2010

48. Brenda C, O'Connell L, Adamson B, et al: A genome-wide camptothecin sensitivity screen identifies a mammalian MMS22L-NFKBIL2 complex required for genomic stability. Mol Cell 40: 645-657: 2010.

49. Piwko W, Olma MH, Held M, et al: RNAi-based screening identifies the Mms22L-Nfkbil2 complex as a novel regulator of DNA replication in human cells. EMBO J 29: 4210-4222, 2010.

50. Lee BJ, Chon KM, Kim YS, et al: Effects of cisplatin, 5-fluorouracil, and radiation on cell cycle regulation and apoptosis. Chemotherapy 51: 103-110, 2005. 